\title{
First report of natural infection of tomato by pepper mild mottle virus in China
}

\author{
Wen Peng Zhou ${ }^{1} \cdot$ Yue Yue $\mathrm{Li}^{1,2} \cdot$ Fan $^{\mathrm{Li}^{1}} \cdot$ Guan Lin $\operatorname{Tan}^{3}$ (D) \\ Received: 10 March 2020 / Accepted: 13 October 2020 / Published online: 2 November 2020 \\ (C) Società Italiana di Patologia Vegetale (S.I.Pa.V.) 2020
}

Keywords PMMoV · Tomato

In June 2016, fern leaf and necrosis symptoms were observed on leaves of three tomato (Solanum lycopersicum) plants in field of Chuxiong autonomous prefecture Yunnan province, China. Total nucleic acids were extracted using a CTAB method from the diseased tomato leaves stored in $-80{ }^{\circ} \mathrm{C}$ and the nucleic acid extracts were tested for the presence of tobamoviruses by RT-PCR with universal primers TobamodF/TobamodR (Li et al. 2018) in 2019. Amplicons of approximately $880 \mathrm{bp}$ were obtained from the three samples, and sequencing results showed pepper mild mottle virus (PMMoV) infecting on the tomato samples. Then the specific primers of several tobamoviruses including PMMoV, tobacco mosaic virus and tomato mosaic virus were used to retest the presence of PMMoV and other tobamoviruses, and PMMoV infection was confirmed with specific primer pair of PMMoVdF (5'-GTAAGAGAAATGATAATAAGGGTTTG -3')/PMMoVdR (5'-CGTTCGCAAATACACGTCAC-3') from the diseased tomato samples. The $720 \mathrm{bp} \mathrm{PMMoV} \mathrm{se-}$ quences obtained from all three samples were identical and deposited in GenBank (Accession No. MN515416). NCBI BLAST search indicated that the nucleotide sequence shared the highest nucleotide sequence identity of $99 \%$ with PMMoV isolate $\mathrm{J}$ (AB000709). To determine the pathogenicity of PMMoV to tomato, healthy tomato plants were inoculated with PMMoV-infectious clone via agrobacterium mediated method. Mild mottle symptom was observed in 2 out of 5 inoculated tomato plants 20 days post inoculation, RT-PCR and sequencing results confirmed the PMMoV infection. To our knowledge this is the first report of natural infection of PMMoV on tomato in China.

Acknowledgement This research was supported by the National Natural Science Foundation of China (31660039) and the Program for Innovative Research Team (in Science and Technology) in University of Yunnan Province (Yunjiaoke 2014-22).

\section{Reference}

Li YY, Tan GL, Lan PX, Zhang AS, Liu Y, Li RH, Li F (2018) Detection of tobamoviruses by RT-PCR using a novel pair of degenerate primers. J Virol Methods 259:122-128

Publisher's note Springer Nature remains neutral with regard to jurisdictional claims in published maps and institutional affiliations.
Guan Lin Tan

gltan@126.com

1 State Key Laboratory for Conservation and Utilization of Bio-Resources in Yunnan, Yunnan Agricultural University, Kunming 650201, China

2 College of Life Science, Luoyang Normal University, Luoyang 471934, China

3 Modern Education Technology Center, Yunnan Agricultural University, Kunming 650201, China 